PROCEEDINGS OF THE

AMERICAN MATHEMATICAL SOCIETY

Volume 137, Number 4, April 2009, Pages 1411-1420

S 0002-9939(08)09650-0

Article electronically published on November 3, 2008

\title{
HYPERBOLIC DIMENSION AND RADIAL JULIA SETS OF TRANSCENDENTAL FUNCTIONS
}

\author{
LASSE REMPE \\ (Communicated by Jane M. Hawkins)
}

\begin{abstract}
We survey the definition of the radial Julia set $J_{r}(f)$ of a meromorphic function (in fact, more generally, any Ahlfors islands map), and give a simple proof that the Hausdorff dimension of $J_{r}(f)$ and the hyperbolic dimension $\operatorname{dim}_{\mathrm{hyp}}(f)$ always coincide.
\end{abstract}

\section{INTRODUCTION}

The study of the measurable dynamics of rational functions is well developed. In particular, it is known $[\mathrm{P}, \mathrm{DU}]$ that a large number of dynamically natural quantities coincide (compare also the survey article [U2]):

(1) The Hausdorff dimension of the radial (also called conical) Julia set $J_{r}(f)$, introduced by Urbański [U1] and McMullen [McM] as the set of points where it is possible to go from small to large scales via univalent iterates. (See Definition 2.5.) We remark that there are a number of different and inequivalent definitions of "conical Julia sets" in the literature; see [P, Appendix 3] for a discussion.

(2) The hyperbolic dimension, defined by Shishikura Sh as the supremum of the Hausdorff dimensions of hyperbolic sets for $f$. (See Definition 2.7.)

(3) The dynamical dimension, defined by Denker and Urbański DU] as the supremum over the Hausdorff dimensions of all ergodic invariant probability measures of positive entropy.

(4) The minimal exponent for which a conformal measure in the sense of Sullivan exists.

In well-controlled cases, these numbers also agree with the Hausdorff dimension of $J(f)$, but it is unknown whether this holds in general (for transcendental functions, this is often false [S3, $\mathrm{UZ}$, and results of Avila and Lyubich $\mathrm{AL}$ also suggest the existence of rational functions for which this equality fails).

In recent years, there have been a large number of cases where the above program was also carried through for transcendental entire and meromorphic functions. (We refer the reader to the survey $[\mathrm{KU}$.) This suggests the question which parts of the general rational theory carry through for arbitrary transcendental meromorphic

Received by the editors December 21, 2007, and, in revised form, May 22, 2008.

2000 Mathematics Subject Classification. Primary 37F35; Secondary 37F10, 30D05.

Key words and phrases. Hyperbolic dimension, radial Julia set, iterated function system, entire function, meromorphic function, Ahlfors islands map, Hausdorff dimension, conformal measure.

The author was supported by EPSRC grant EP/E017886/1. 
functions. The purpose of this note is to observe the following (where $\operatorname{dim}_{H}$ denotes Hausdorff dimension).

1.1. Theorem (Equality of hyperbolic and radial dimensions). For a nonconstant, nonlinear meromorphic function $f: \mathbb{C} \rightarrow \hat{\mathbb{C}}$, we have $\operatorname{dim}_{\text {hyp }}(f)=\operatorname{dim}_{H}\left(J_{r}(f)\right)$.

Remark. In fact, we prove this theorem even more generally for the class of Ahlfors islands maps studied by Epstein [E1] and Oudkerk [0] (see Definition 2.1 below).

This equality is usually established using conformal measures of the correct exponent for the function $f$. However, the construction of such measures seems more difficult in the transcendental case, and, in currently known cases, uses additional dynamical and function-theoretic assumptions. Our main observation is that it is possible to avoid this step and construct a suitable hyperbolic set directly from the radial Julia set. Even for rational functions, this argument (though not the theorem) seems to be new. We believe the construction is interesting due to its simplicity and wide generality.

Stallard has shown that the Julia set of a transcendental meromorphic function can have any Hausdorff dimension in the interval $(0,2]$, and that for a transcendental entire function this dimension can be any number in the interval $(1,2]$. She also proved [S1] that the hyperbolic dimension is always strictly greater than 0 (the proof carries over directly to Ahlfors islands maps, and we sketch it in Corollary 2.11 below). It is not known in general whether the Hausdorff dimension of the Julia set of an entire transcendental function can equal the lower bound 1, but for functions with a logarithmic singularity over infinity, this is not the case S2, RS, BRS. Recently, Barański, Karpińska and Zdunik [BKZ] extended this result to show also that the hyperbolic dimension of such a function is strictly larger than one. We discuss a minor generalization of this fact in Theorem A.1.

Structure of the article. In Section 2, we review a number of definitions which do not explicitly appear in the literature in this generality. The beautiful concepts of finite-type and Ahlfors islands maps, both due to Adam Epstein, have appeared only in the unpublished references [E2, E1, O], which are not widely available, so we take the opportunity to introduce them here. We also define radial Julia sets, whose well-known definition in the rational case carries over verbatim but does not seem to have been formally stated in the transcendental setting. We also review the definition of hyperbolic dimension, and for the reader's convenience include a short summary on an elementary type of conformal iterated function system which will be used to construct hyperbolic sets. Finally, we prove Theorem 1.1 in Section 3 , and discuss hyperbolic dimension in logarithmic tracts in an appendix.

Notation. Throughout the rest of this article, $X$ is a compact Riemann surface and $W \subset X$ is a nonempty open subset. A reader who is interested only in the classical cases may wish to assume that $W=\mathbb{C}$ and $X=\hat{\mathbb{C}}$ (for transcendental meromorphic functions) or $W=X=\hat{\mathbb{C}}$ (for rational functions) and skip the subsection on Ahlfors islands maps in Section 2, All distances, balls, derivatives etc. are considered with respect to the natural conformal metric on the Riemann surface $X$ (i.e., the unique metric of constant curvature $-1,0$ or 1 ). Since $X$ is compact, for sufficiently small $\delta>0$, any disk $\mathbb{D}_{\delta}\left(z_{0}\right)=\left\{z \in X: \operatorname{dist}\left(z_{0}, z\right)<\delta\right\}$ is simply connected with Jordan curve boundary. Recall that by Radò's theorem, $X$ is second countable, so there is a countable base for the topology of $X$ consisting of 
such simply connected balls. If $A \subset X$ and $a \in A$, then we denote by $\operatorname{Comp}_{a}(A)$ the connected component of $A$ containing $a$. Finally, we remark that "meromorphic" for us always includes "entire" as a special case.

\section{Definitions}

Ahlfors islands maps. The class of Ahlfors islands maps was suggested by Adam Epstein [E1] as a generalization of both general transcendental meromorphic functions and his "finite-type maps" (see below), and studied in greater detail by him and Richard Oudkerk [O]. This class provides a general setting for the study of transcendental dynamics. We remark that a similar condition for a restricted class of maps (those defined outside a small subset of the Riemann sphere) was considered by Baker, Domínguez and Herring in $\mathrm{BDH}]$.

2.1. Definition (Ahlfors Islands maps). Let $W \subset X$ be open and nonempty. We say that a nonconstant holomorphic function $f: W \rightarrow X$ is an Ahlfors islands map if there is a finite number $k$ satisfying the following condition (known as the "islands property").

Let $V_{1}, \ldots, V_{k} \subset X$ be Jordan domains with pairwise disjoint closures, and let $U \subset X$ be open and connected with $U \cap \partial W \neq \emptyset$. Then for every component $U_{0}$ of $U \cap W$, there is $i \in\{1, \ldots, k\}$ such that $f$ has a simple island over $V_{i}$ in $U_{0}$. (That is, there is a domain $G \subset U_{0}$ such that $f: G \rightarrow V_{i}$ is a conformal isomorphism.)

An Ahlfors islands map is elementary if $W=X$ and $f: W \rightarrow X$ is a conformal isomorphism. The set of all nonelementary Ahlfors islands maps from $W$ to $X$ is denoted $\mathcal{A}(W, X)$. We also set

$$
\begin{aligned}
\mathcal{A}(X) & :=\bigcup\{\mathcal{A}(W, X): W \subset X \text { open, nonempty }\} \text { and } \\
\mathcal{A} & :=\bigcup\{\mathcal{A}(X): X \text { is a compact Riemann surface }\}
\end{aligned}
$$

The class of Ahlfors islands maps is closed under composition. We note that nonconstant rational functions are Ahlfors islands maps by definition. The name of this class is derived from the classical five islands theorem [B, Theorem A.2], which implies that every transcendental meromorphic function $f: \mathbb{C} \rightarrow \hat{\mathbb{C}}$ is an element of $\mathcal{A}(\mathbb{C}, \hat{\mathbb{C}})$ (with $k=5$ ). We shall make use of the normal families version [B. Theorem A.1] of this theorem: if $\mathcal{F}$ is a family of meromorphic functions which have no simple islands over given domains $V_{1}, \ldots, V_{5}$ as above, then $\mathcal{F}$ is normal.

Another interesting subclass of $\mathcal{A}(W, X)$ is that of finite-type maps, first introduced in [E2, Chapter 3], which provides a natural generalization of the dynamics of rational functions and meromorphic functions with finitely many singular values. A nonconstant analytic function $f: W \rightarrow X$ is a finite-type map if it has only finitely many singular values in $X$, and furthermore there are no isolated removable singularities in $\partial W$.

2.2. Theorem (Epstein). If $f: W \rightarrow X$ is a finite-type map, then $f$ is an Ahlfors islands map.

There are many examples of Ahlfors islands maps which do not fall into either of the previous two classes. For example, a "Valiron function" is an analytic map $f: \mathbb{D} \rightarrow \mathbb{C}$ which is unbounded in $\mathbb{D}$, but bounded on a "spiral", i.e., on a curve $\gamma(t)=r(t) \cdot e^{2 \pi i \vartheta(t)}$ with $r(t) \nearrow 1$ and $|\vartheta(t)| \rightarrow \infty$. Every such function is an Ahlfors islands map (see [BR2, Remark in Section 4]); indeed, these functions are 
not normal near any point of $\partial \mathbb{D}$, and the result follows by the normal families version of the Ahlfors five islands theorem. For the same reason, other functions for which normality fails in this way, e.g. the functions in [BR2, Theorem 1.3], also belong to the class of Ahlfors islands maps. (Compare also $[\mathrm{RR}$. .)

The Fatou set $F(f) \subset X$ of an Ahlfors islands map $f: W \rightarrow X$ is now defined as usual, except that, in addition to those points which have a neighborhood where all iterates are defined and form a normal family, it will also include all points with $f^{j}(z) \in X \backslash \bar{W}$ for some $j$. Thanks to the Ahlfors islands property, Baker's proof of the density of repelling periodic points in the Julia set $J(f):=X \backslash F(f)$ goes through as usual, and $J(f)$ has all the standard properties.

2.3. Theorem (Baker, Epstein). Let $f \in \mathcal{A}(W, X)$. Then $J(f)$ is a nonempty perfect subset of $X$, and repelling periodic points of $f$ are dense in $J(f)$. Furthermore, if $J(f) \neq X$, then $J(f)$ has empty interior.

Note that $J(f) \neq \emptyset$ is trivial by definition as soon as $\partial W \neq \emptyset$, while $\partial W=\emptyset$ means that we are in the well-known case of a rational self-map of the Riemann sphere or of a self-map of the torus. The key fact in the proof of the density of repelling periodic points is the following lemma, which we will use below, and whose proof we sketch for completeness. The remaining claims in Theorem 2.3 follow in the usual manner.

2.4. Lemma (Islands near the Julia set). Let $f \in \mathcal{A}(W, X)$. Then there is a number $k$ with the following property: if $V_{1}, \ldots, V_{k} \subset X$ are Jordan domains with pairwise disjoint closures, then there is some $V_{j}$ such that every open set $U$ with $U \cap J(f) \neq \emptyset$ contains a simple island of $f^{n}$ over $V_{j}$ for some $n$.

Proof. Let $k \geq 5$ be as in the islands property. First suppose that $\partial W \neq \emptyset$; then by the islands property, $f$ has infinitely many simple islands over some $V_{j}$; in particular, we can pick $k$ such islands $G_{1}, \ldots, G_{k}$.

If $\partial W=\emptyset$, then $f$ must be either a rational function or a self-map of the torus; in either case, it is easy to see that there is some $V_{j}$ and $k$ domains $G_{1}, \ldots, G_{k}$ which are simple islands of some iterates of $f$. (For example, this follows by using the fact that $J(f) \neq \emptyset$ and the normal families version of the Ahlfors five islands theorem.)

Now let $U$ be as in the statement of the lemma. If all iterates of $f$ are defined on $U$, then the normal families version of the Ahlfors five islands theorem implies the existence of a simple island of some iterate of $f$ over one of the domains $G_{1}, \ldots, G_{k}$, which is hence also an iterated island over the original domain $V_{j}$.

Otherwise, there is some $z_{0} \in U$ with $f^{n}\left(z_{0}\right) \in \partial W$. By shrinking $U$, if necessary, we may assume that $\left.f^{n}\right|_{U}$ is a proper map with no critical points except possibly $z_{0}$. By the islands property, $f^{n}(U)$ contains a simple island of $f$ over one of the $G_{1}, \ldots, G_{k}$, which then pulls back to a simple island of $f^{n+2}$ over $V_{j}$, as desired.

Remark. Epstein's original definition of finite-type and Ahlfors islands maps allows $X$ to be the disjoint union of several compact Riemann surfaces; we have restricted ourselves to the connected case for simplicity. We also remark that Oudkerk [0] has suggested a slightly modified definition of Ahlfors islands maps which is equivalent to that given above but is somewhat more natural with respect to composition. 
For finite-type maps, Epstein proves the absence of Baker and wandering domains as well as the finiteness of nonrepelling cycles. We refer the reader to the thesis E2 and the manuscript E1 for further information.

\section{Radial Julia sets.}

2.5. Definition (Radial Julia sets). Let $f \in \mathcal{A}(W, X)$. The radial Julia set $J_{r}(f)$ is the set of all points $z \in J(f)$ with the following property: all forward images of $z$ are defined, and there is some $\delta>0$ such that, for infinitely many $n \in \mathbb{N}$, the disk $\mathbb{D}_{\delta}\left(f^{n}(z)\right)$ can be pulled back univalently along the orbit of $z$. (That is, $f^{n}: \operatorname{Comp}_{z}\left(f^{-n}\left(\mathbb{D}_{\delta}\left(f^{n}(z)\right)\right)\right) \rightarrow \mathbb{D}_{\delta}\left(f^{n}(z)\right)$ is a conformal isomorphism.)

Recall that all disks and distances are understood with respect to the metric on the compact surface $X$ (i.e., the spherical metric in the case of rational, entire or meromorphic functions, where $X=\hat{\mathbb{C}})$. We remark that, if $z \in J(f)$ and $\limsup \operatorname{dist}\left(f^{n}(z), \mathcal{P}(f)\right)>0$, where $\mathcal{P}(f)$ denotes the postsingular set of $f$, then $z \in J_{r}(f)$.

2.6. Lemma and Definition (Disks of Univalence). Let $z_{0} \in J_{r}(f)$. Then there exists a disk $D=\mathbb{D}_{\delta}\left(w_{0}\right) \subset X$ with the following properties.

(1) Both $D$ and $\mathbb{D}_{2 \delta}\left(w_{0}\right)$ are simply connected.

(2) For infinitely many $n, f^{n}\left(z_{0}\right) \in D$ and $\mathbb{D}_{2 \delta}\left(w_{0}\right)$ pulls back univalently to $z_{0}$ under $f^{n}$.

In particular, if $n$ is as above and $U$ is the component of $f^{-n}(D)$ containing $z_{0}$, then

$$
\vartheta:=\sup _{z \in U}\left\|D f^{n}(z)\right\|<\infty \quad \text { and } \quad U \subset \mathbb{D}_{C / \vartheta}\left(z_{0}\right)
$$

(Here $C$ depends only on $X$.) Furthermore, $\vartheta \rightarrow \infty$ as $n \rightarrow \infty$.

We will call such a disk $D$ a disk of univalence for $z_{0}$.

Proof. Let $\delta^{\prime}$ be the constant from the definition of $J_{r}(f)$. Let $w_{0}$ be a limit point of a sequence $z_{n}=f^{n}\left(z_{0}\right)$ of iterates for which $\mathbb{D}_{\delta^{\prime}}\left(z_{n}\right)$ can be pulled back univalently. Then we set $D:=\mathbb{D}_{\delta}\left(w_{0}\right)$, where $\delta<\delta^{\prime} / 2$ is sufficiently small so that $D$ and $\mathbb{D}_{2 \delta}\left(w_{0}\right)$ are simply connected.

The final claim follows from the Koebe distortion theorem.

Hyperbolic dimension. Hyperbolic dimension was introduced by Shishikura in his proof $\mathrm{Sh}$ ] that the boundary of the Mandelbrot set has Hausdorff dimension two.

2.7. Definition (Hyperbolic dimension). Let $f \in \mathcal{A}(W, X)$. A hyperbolic set is a compact, forward-invariant set $K \subset W$ such that $\left\|\left.D f^{n}\right|_{K}\right\|>\lambda>1$ for some $n$ and $\lambda$.

The hyperbolic dimension of $f$ is the supremum of $\operatorname{dim}_{H}(K)$ over all hyperbolic sets.

The following fact is well-known (compare [RvS, Lemma 3.4]).

2.8. Lemma (Hyperbolic sets and the radial Julia set). Any hyperbolic set is contained in $J_{r}(f)$. Conversely, any compact and forward-invariant subset of $J_{r}(f)$ is hyperbolic.

In particular, the hyperbolic dimension $\operatorname{dim}_{\text {hyp }}(f)$ is no larger than the Hausdorff dimension of $J_{r}(f)$. 
Conformal iterated function systems. Hyperbolic sets are usually obtained by constructing a conformal iterated function system consisting of iterates of the function $f$. We review the basic case of the theory for the reader's convenience.

2.9. Definition (Elementary conformal IFS). Let $V \subset X$ be simply connected. Also let $U_{1}, \ldots, U_{m} \subsetneq V$ be pairwise disjoint simply connected domains with $\overline{U_{j}} \subset$ $V$, and let $g_{j}: V \rightarrow U_{j}$ be conformal isomorphisms. Then we say that $\left(g_{j}\right)$ is an elementary conformal iterated function system.

The limit set of the system $\left(g_{j}\right)$ is the set

$$
L:=\bigcap_{n \geq 0} \bigcup_{\left(j_{1}, \ldots, j_{n}\right)} g_{j_{n}}\left(\ldots\left(g_{j_{1}}(V)\right)\right) .
$$

(By the Schwarz lemma, the maps $g_{j}$ are contractions, and hence $L$ is a compact, totally disconnected set.)

In the well-known case where all $g_{j}$ are affine similarities, the Hausdorff dimension of the limit set is the unique value $t$ with

$$
\sum\left(\lambda_{j}\right)^{t}=1
$$

where $\left|g_{j}^{\prime}\right| \equiv \lambda_{j}<1[\mathrm{Fa}$. We will use the following lemma in the general case.

2.10. Lemma (Hausdorff dimension of limit sets). Suppose that $L$ is the limit set of a conformal iterated function system $\left(g_{j}\right)$ as above. Set $\lambda_{j}:=\inf _{z \in V}\left\|D g_{j}(z)\right\|$, and suppose that $\sum_{j} \lambda_{j}{ }^{t} \geq 1$. Then $\operatorname{dim}_{H}(L) \geq t$.

In fact, the Hausdorff dimension of the limit set can be computed exactly by generalizing the formula (11) to the more general setting as follows. We set

$$
P(t):=\lim _{n \rightarrow \infty} \frac{1}{n} \log \left(\sum_{\left(j_{1}, \ldots, j_{n}\right)}\left\|D\left(g_{j_{1}} \circ \cdots \circ g_{j_{n}}\right)\left(z_{0}\right)\right\|^{t}\right),
$$

where $z_{0} \in V$ is some base point. (The existence of the limit and its independence from $z_{0}$ follow from the Koebe distortion theorem.)

Then the Hausdorff dimension $t$ is the unique zero of the strictly decreasing function $P(t)$, usually called the "pressure function". (For a proof, in the more general setting of infinite conformal iterated function systems in any dimension, see [MU].)

We obtain the above lemma as an obvious corollary, since the terms in the limit in (2) are bounded from below by

$$
\frac{1}{n} \log \left(\left(\sum \lambda_{j}^{t}\right)^{n}\right)=\log \left(\sum \lambda_{j}^{t}\right) .
$$

2.11. Corollary (Hyperbolic dimension of Ahlfors islands maps). Let $f \in \mathcal{A}(W, X)$ be a nonelementary Ahlfors islands map. Then $\operatorname{dim}_{\text {hyp }}(f)>0$.

Proof. Let $k$ be as in Lemma 2.4 and pick $k$ pairwise disjoint Jordan domains $U_{1}, \ldots, U_{k}$, each of which intersects the Julia set. Then for at least one of these domains, say $U=U_{j}$, there are simple islands of some iterate of $f$ over $U$ near every point of the Julia set. Using the fact that $J(f)$ is perfect, we can thus find two (in fact infinitely many) domains $V_{1}, V_{2} \Subset U$, with $\overline{V_{1}} \cap \overline{V_{2}}=\emptyset$, such that some iterate of $f$ maps $V_{j}$ conformally to $U$. The corresponding inverse branches $g_{j}: U \rightarrow V_{j}$ then form an elementary conformal iterated function system, and the 
limit set $L$ of this system has Hausdorff dimension strictly greater than 0 . Clearly $L$ together with its finitely many forward images is a hyperbolic set for $f$, and the claim follows.

Hausdorff dimension and backward invariance. We include a proof of the following simple lemma.

2.12. Observation (Hausdorff dimension of backward invariant sets). Let $f \in$ $\mathcal{A}(W, X)$ be a nonelementary Ahlfors islands map, and let $B \subset X$ be backward invariant under $f$, i.e. $f^{-1}(B) \subset B$.

If $U \subset X$ is an open set intersecting $J(f)$, then $\operatorname{dim}_{H}(B \cap U)=\operatorname{dim}_{H}(B)$.

Proof. It follows easily from the definition of Hausdorff dimension that there is a sequence $D_{1}, D_{2}, \ldots$ of Jordan disks with pairwise disjoint closures such that $\operatorname{dim}_{H}\left(B \cap D_{j}\right) \rightarrow \operatorname{dim}_{H}(B)$.

If $U$ contains some point where $f^{k}$ is undefined for some $k \geq 0$, then it follows from the Ahlfors islands property that $U$ contains a simple island of $f^{k}$ over $D_{j}$ for infinitely many $j$. Otherwise, this follows from the usual five islands theorem for (non)normal families. So in either case (since conformal maps preserve Hausdorff dimension), we have $\operatorname{dim}_{H}(B \cap U) \geq \operatorname{dim}_{H}\left(B \cap D_{j}\right)$ for infinitely many $j$, which proves the claim.

\section{Hyperbolic Dimension and RAdial Julia SETS}

3.1. Theorem. Let $f \in \mathcal{A}(W, X)$ be any nonelementary Ahlfors islands map. Then $\operatorname{dim}_{\text {hyp }}(f)=\operatorname{dim}_{H}\left(J_{r}(f)\right)$.

Proof. The inequality " $\leq$ " follows from Lemma 2.8. So set $d:=\operatorname{dim}_{H}\left(J_{r}(f)\right)$ and let $\varepsilon>0$. We shall show that $\operatorname{dim}_{\text {hyp }}(f) \geq d^{\prime}:=d-\varepsilon$ by constructing a hyperbolic set of dimension at least $d^{\prime}$.

Pick a countable basis of the topology of $X$ consisting of simply connected disks $D_{i}$. Let $J_{r}\left(D_{i}\right)$ be the set of points in $J_{r}(f)$ for which $D_{i}$ is a disk of univalence as defined in Lemma 2.6. Note that, if $z \in J_{r}\left(D_{i}\right)$ and $w \in W$ with $f(w)=z$, then either $w \in J_{r}\left(D_{i}\right)$ or $w$ is a critical point. So $J_{r}\left(D_{i}\right)$ is the union of a backward invariant set and a countable set, and hence all nonempty open subsets of $J_{r}\left(D_{i}\right)$ have the same Hausdorff dimension by Observation 2.12.

Since $J_{r}(f)$ is the countable union of the $J_{r}\left(D_{i}\right)$, we can find an index $i$ such that $J_{r}\left(D_{i}\right)$ has Hausdorff dimension greater than $d^{\prime}$. Set $D:=D_{i}$ and let $A:=$ $J_{r}(D) \cap D$; then $\operatorname{dim}_{H}(A)>d^{\prime}$ by the above. Hence, we can pick $\delta>0$ such that any covering of $A$ by sets $U_{j}$ of diameter at most $\delta$ has

$$
\sum_{j} \operatorname{diam}\left(U_{j}\right)^{d^{\prime}}>(10 \cdot C)^{d^{\prime}}
$$

where $C$ is the constant from Lemma 2.6 .

Now, for every point $a \in A$, pick a univalent pullback $U_{a}$ of $V$ around $a$ as in Lemma 2.6 such that

$$
\vartheta_{a}:=\sup _{z \in U_{a}}\left\|D f^{n}(z)\right\|>\frac{10 C}{\delta}
$$

and $\overline{U_{a}} \subset V$. Then $A$ is covered by the open balls $\mathbb{D}_{C / \vartheta_{a}}(a)$, and by a standard covering theorem (see [M, Theorem 2.1] or [Fe, Theorem 2.84 and corollaries]), we can pick a subsequence $a_{j}$ such that the balls $\mathbb{D}_{C / \vartheta_{j}}\left(a_{j}\right)$ are disjoint (where $\left.\vartheta_{j}=\vartheta_{a_{j}}\right)$ while $A \subset \bigcup \mathbb{D}_{5 C / \vartheta_{j}}\left(a_{j}\right)$. 
By the condition on the size of $\vartheta_{a}$, these balls have diameter $10 C / \vartheta_{j}<\delta$. So by choice of $\delta$, we have

$$
\sum_{j=1}^{\infty}\left(\frac{10 C}{\vartheta_{j}}\right)^{d^{\prime}}>(10 C)^{d^{\prime}} .
$$

If we pick $N$ sufficiently large, set $\lambda_{j}:=1 / \vartheta_{j}$ and consider the elementary conformal iterated function system given by the branches $g_{j}$ of $f^{-n_{j}}$ which take $D$ to $U_{j}$, we have

$$
\sum_{j=1}^{N} \lambda_{j}^{d^{\prime}}>1
$$

and hence the limit set $L$ of this system satisfies $\operatorname{dim}_{H}(L) \geq d^{\prime}$ by Lemma 2.10. We have $L \subset A \subset J_{r}(f)$ by construction, and $L$ together with finitely many forward images forms a compact, forward-invariant set, which is hence a hyperbolic set for $f$.

\section{APPENDIX: HyperboliC DIMENSION IN LOGARITHMIC TRACTS}

If $U \subset \mathbb{C}$ is an unbounded Jordan domain, and $f: U \rightarrow\{|z|>R\}$ (where $R>0$ ) is a universal covering, then Barański, Karpińska and Zdunik [BKZ] show that there is a hyperbolic set $K \subset U$ for $f$ with $\operatorname{dim}_{H}(K)>1$. We remark that this carries over to a slightly more general setting where the asymptotic curves only accumulate on the asymptotic value:

A.1. Theorem (Hyperbolic dimension and logarithmic tracts). Let $X$ be a compact Riemann surface, let $D \subset X$ be a Jordan domain, and let $a \in D$.

Suppose that $G \subset X \backslash\{a\}$ is a simply connected domain, and that

$$
f: G \rightarrow D \backslash\{a\}
$$

is a universal covering map. Suppose furthermore that there is a sequence $\left(z_{n}\right)$ in $G$ with $z_{n} \rightarrow a$ and $f\left(z_{n}\right) \rightarrow a$.

Then $G$ contains a hyperbolic set for $f$ of dimension greater than one.

In the setting of [BKZ, one has the stronger property that $z_{n} \rightarrow a$ whenever $f\left(z_{n}\right) \rightarrow a$ in $G$. We note that it is easy to construct an Ahlfors islands function with a logarithmic tract which does not satisfy this stronger condition, but to which the theorem applies. For example, we can create such a function by modifying the elliptic modular function $j: \mathbb{D} \rightarrow \hat{\mathbb{C}} \backslash\{0,1, \infty\}$. Let $U \subset \mathbb{C}$ be a simply connected domain which has a prime end $p$ whose set $\Pi(p)$ of principal points is nontrivial and contains $\infty$. Let $\zeta \in \partial \mathbb{D}$ be a point such that $j$ has radial limit $\infty$ at $\mathbb{D}$, and let $\varphi: \mathbb{D} \rightarrow U$ be a Riemann map under which $\zeta$ corresponds to the prime end $p$. Then $f:=j \circ \varphi^{-1}$ is a finite-type map (and hence an Ahlfors islands map by Theorem 2.2), and has an asymptotic curve which accumulates on all principal points of $p$. So $f$ satisfies the hypotheses of Theorem A.1, where $a=\infty, D=\hat{\mathbb{C}} \backslash \overline{\mathbb{D}_{2}(0)}$ and $G$ is the component of $f^{-1}(D)$ which contains a tail of this asymptotic curve.

For a perhaps more natural example, suppose that $f: \mathbb{D} \rightarrow \mathbb{C}$ is a Valiron function (as discussed in Section 2) which has a logarithmic asymptotic value over $\infty$. (Such a function can be constructed by the same technique as in [BR1, Example 1].) Then the asymptotic curve itself spirals in to the boundary. Postcomposing $f$ with a suitable Möbius transformation which takes $\infty$ to the unit circle gives rise to an 
Ahlfors islands map with the aforementioned property. For many further examples of Ahlfors islands maps with logarithmic singularities, see [RR, Theorem 1.3].

The proof in BKZ, whose underlying ideas can be traced to Stallard's original argument from [S2], essentially also proves our more general version. We will briefly explain one detail in the proof which differs slightly in our setting.

Consider an annulus $A$ centered at $a$ (in some fixed local coordinates), of sufficiently large modulus and sufficiently close to $a$. Then, in the setting of [BKZ, the preimage of this annulus under $f$ is an infinite strip $S$ tending to $a$ in both directions. By basic estimates on the hyperbolic metric, $S$ must contain points on the outside of the annulus, and hence crosses $A$. This fact allows Barański, Karpińska and Zdunik to construct an iterated function system (in logarithmic coordinates) whose limit set has Haudorff dimension greater than one.

With the more general assumptions in Theorem A.1, it is no longer true that the strip $S$ must tend to $a$. (Recall the examples discussed above.) However, $S$ still crosses the annulus $A$ at least once, and possibly several times. (This follows from the assumptions on $f$ and well-known results of geometric function theory, e.g. the fact that radial limit points of a conformal map are dense in the boundary of its image.) The remainder of the proof goes through exactly as in BKZ; we refer the reader to this article for further details.

\section{ACKNowledgments}

I would like to thank Boguslawa Karpińska, Janina Kotus, Feliks Przytycki, Michał Rams, Gwyneth Stallard and Anna Zdunik for interesting discussions.

\section{REFERENCES}

AL. Artur Avila and Mikhail Lyubich, Hausdorff dimension and conformal measures of Feigenbaum Julia sets, J. Amer. Math. Soc. 21 (2008), no. 2, 305-363. MR2373353

BDH. I. N. Baker, P. Domínguez, and M. E. Herring, Dynamics of functions meromorphic outside a small set, Ergodic Theory Dynam. Systems 21 (2001), no. 3, 647-672. MR.1836425 (2002e:37058)

BKZ. Krzysztof Barański, Boguslawa Karpińska, and Anna Zdunik, Hyperbolic dimension of Julia sets of meromorphic maps with logarithmic tracts, preprint, 2007, arXiv.org/abs/0711.2672v1.

BR1. Karl F. Barth and Philip J. Rippon, Exceptional values and the MacLane class A, Bergman spaces and related topics in complex analysis, Contemp. Math., vol. 404, Amer. Math. Soc., Providence, RI, 2006, pp. 41-52. MR2244003 (2007k:30054)

BR2. _ Extensions of a theorem of Valiron, Bull. London Math. Soc. 38 (2006), no. 5, 815-824. MR2268366 (2007g:30052)

B. Walter Bergweiler, A new proof of the Ahlfors five islands theorem, J. Anal. Math. 76 (1998), 337-347. MR1676971 (99m:30032)

BRS. Walter Bergweiler, Philip J. Rippon, and Gwyneth M. Stallard, Dynamics of meromorphic functions with direct or logarithmic singularities, preprint, 2007, arXiv.org/abs/0704.2712, Proc. London Math. Soc. 97 (2008), no. 2, 368-400.

DU. M. Denker and M. Urbański, On Sullivan's conformal measures for rational maps of the Riemann sphere, Nonlinearity 4 (1991), no. 2, 365-384. MR1107011 (92f:58097)

E2. Adam L. Epstein, Towers of finite type complex analytic maps, Ph.D. thesis, City Univ. of New York, 1995. Available at http://pcwww.liv.ac.uk/ lrempe/adam/thesis.pdf.

E1. D D D _ Damics of finite type complex analytic maps I: Global structure theory, manuscript.

Fa. Kenneth Falconer, Fractal geometry, Mathematical foundations and applications, John Wiley \& Sons Ltd., Chichester, 1990. MR.1102677 (92j:28008) 
Fe. Herbert Federer, Geometric measure theory, Die Grundlehren der Mathematischen Wissenschaften, Band 153, Springer-Verlag New York Inc., New York, 1969. MR0257325 (41:1976)

KU. Janina Kotus and Mariusz Urbański, Fractal measures and ergodic theory of transcendental meromorphic functions, Transcendental Dynamics and Complex Analysis (P. Rippon and G. Stallard, eds.), London Math. Soc. Lecture Note Ser., vol. 348, Cambridge Univ. Press, 2008.

M. Pertti Mattila, Geometry of sets and measures in Euclidean spaces, Fractals and rectifiability, Cambridge Studies in Advanced Mathematics, vol. 44, Cambridge University Press, Cambridge, 1995. MR1333890 (96h:28006)

MU. R. Daniel Mauldin and Mariusz Urbański, Dimensions and measures in infinite iterated function systems, Proc. London Math. Soc. (3) 73 (1996), no. 1, 105-154. MR1387085 (97c:28020)

McM. Curtis T. McMullen, Hausdorff dimension and conformal dynamics. II. Geometrically finite rational maps, Comment. Math. Helv. 75 (2000), no. 4, 535-593. MR 1789177 (2001m:37089)

O. Richard Oudkerk, Iteration of Ahlfors and Picard functions which overflow their domains, manuscript.

P. Feliks Przytycki, Conical limit set and Poincaré exponent for iterations of rational functions, Trans. Amer. Math. Soc. 351 (1999), no. 5, 2081-2099. MR1615954 (99h:58110)

RR. Lasse Rempe and Philip J. Rippon, Exotic Baker and wandering domains for Ahlfors islands functions, preprint (2008).

RvS. Lasse Rempe and Sebastian van Strien, Absence of line fields and Mañé's theorem for nonrecurrent transcendental functions, preprint, 2007, arXiv:0802.0666, submitted for publication.

RS. Philip J. Rippon and Gwyneth M. Stallard, Dimensions of Julia sets of meromorphic functions with finitely many poles, Ergodic Theory Dynam. Systems 26 (2006), no. 2, 525-538. MR 2218773 (2007c:37051)

Sh. Mitsuhiro Shishikura, The boundary of the Mandelbrot set has Hausdorff dimension two, Astérisque (1994), no. 222, 7, 389-405, Complex analytic methods in dynamical systems (Rio de Janeiro, 1992). MR.1285397 (96d:58118)

S1. Gwyneth M. Stallard, The Hausdorff dimension of Julia sets of meromorphic functions, J. London Math. Soc. (2) 49 (1994), no. 2, 281-295. MR1260113 (95b:58127)

S2. The Hausdorff dimension of Julia sets of entire functions. II, Math. Proc. Cambridge Philos. Soc. 119 (1996), no. 3, 513-536. MR1357062(97a:58157)

S3. The Hausdorff dimension of Julia sets of hyperbolic meromorphic functions, Math. Proc. Cambridge Philos. Soc. 127 (1999), no. 2, 271-288. MR1705459(2001e:37060)

U1. Mariusz Urbański, On some aspects of fractal dimensions in higher dimensional dynamics, Proceedings of the workshop "Problems in higher dimensional dynamics", preprint SFB 170 Göttingen, vol. 3, 1995, pp. 18-25.

U2. Mariusz Urbański, Measures and dimensions in conformal dynamics, Bull. Amer. Math. Soc. (N.S.) 40 (2003), no. 3, 281-321 (electronic). MR1978566(2004f:37063)

UZ. Mariusz Urbański and Anna Zdunik, The finer geometry and dynamics of the hyperbolic exponential family, Michigan Math. J. 51 (2003), no. 2, 227-250. MR1992945 (2004d:37068)

Department of Mathematical Sciences, University of Liverpool, Liverpool L69 7ZL, UNITED KINGDOM

E-mail address: 1.rempe@liverpool.ac.uk 